\title{
On Hölder regularity for elliptic equations of non-divergence type in the plane
}

\author{
Albert BAERnStein II AND LeOnid V. KOVALEV
}

\begin{abstract}
This paper is concerned with strong solutions of uniformly elliptic equations of non-divergence type in the plane. First, we use the notion of quasiregular gradient mappings to improve Morrey's theorem on the Hölder continuity of gradients of solutions. Then we show that the Gilbarg-Serrin equation does not produce the optimal Hölder exponent in the considered class of equations. Finally, we propose a conjecture for the best possible exponent and prove it under an additional restriction.
\end{abstract}

Mathematics Subject Classification (2000): 35B65 (primary); 30C62, 35J15 (secondary).

\section{Introduction and notation}

Let $\Omega$ be a domain in $\mathbb{R}^{2}$, which will be henceforth identified with the complex plane $\mathbb{C}$. We consider the following second-order equation in non-divergence form

$$
\operatorname{Tr}\left(A D^{2} u\right)=a u_{x x}+2 b u_{x y}+c u_{y y}=0 \quad \text { a.e. in } \Omega,
$$

where $A=\left(\begin{array}{ll}a & b \\ b & c\end{array}\right)$ is a symmetric $2 \times 2$ matrix with measurable real coefficients and $D^{2} u$ is the Hessian matrix of $u$. In what follows it is assumed that $A$ is uniformly elliptic, that is,

$$
|\xi|^{2} / \sqrt{K} \leq\langle A(z) \xi, \xi\rangle \leq \sqrt{K}|\xi|^{2}, \quad \forall \xi \in \mathbb{R}^{2}, \text { a.e. } z \in \Omega
$$

for some constant $K \in[1, \infty)$. Kenig's survey [26] is an excellent general reference for such equations.

A key feature of solutions of many linear and nonlinear elliptic PDE is a "uniform spread" of the eigenvalues of the Hessian matrix [12, 2.2]. In fact, once this property is established, the regularity of solutions can be studied without any further references to the PDE itself [12, Ch. 4]. For this reason, our results concerning the linear equation (1.1) are applicable to some nonlinear PDE as well. See [8, 9, 10, 23] for examples of such equations. 
A real-valued function $u \in W_{\mathrm{loc}}^{2,2}(\Omega)$ is called a strong solution of equation (1.1) if it satisfies (1.1) a.e. in $\Omega$. Strictly speaking, $u$ should be called an $L^{2}$-strong solution, but we are not going to consider $L^{p}$-strong solutions for $p \neq 2$. The main reason is that the Dirichlet problem for $(1.1)$ has a unique $L^{2}$-strong solution $[9,49]$. Furthermore, the class of $L^{p}$-strong solutions does not depend on $p$ when $p$ lies between $2 K /(K+1)$ and $2 K /(K-1)$ [2, 4, 6, 39]. Finally, for values of $p$ outside of this interval, $L^{p}$-strong solutions can be somewhat pathological $[3,4,11,25]$. We therefore restrict our attention to the strong solutions whose second derivatives are locally square integrable.

Every strong solution of (1.1) belongs to the Hölder class $C_{\mathrm{loc}}^{1,1 / K}(\Omega)$. This result can be found in the seminal work of Morrey [34] or in [17, 37] and Ch. 12 of [20]. Safonov [46] proved that the validity of Morrey's estimates distinguishes two-dimensional elliptic equations from their analogues in higher dimensions.

Let us introduce the complex differential operators

$$
\begin{gathered}
\frac{\partial}{\partial z}=\frac{1}{2}\left(\frac{\partial}{\partial x}-i \frac{\partial}{\partial y}\right)=\frac{e^{-i \varphi}}{2}\left(\frac{\partial}{\partial r}-\frac{i}{r} \frac{\partial}{\partial \varphi}\right) ; \\
\frac{\partial}{\partial \bar{z}}=\frac{1}{2}\left(\frac{\partial}{\partial x}+i \frac{\partial}{\partial y}\right)=\frac{e^{i \varphi}}{2}\left(\frac{\partial}{\partial r}+\frac{i}{r} \frac{\partial}{\partial \varphi}\right) ;
\end{gathered}
$$

where $z=x+i y=r e^{i \varphi}, x, y, r, \varphi \in \mathbb{R}$.

Suppose that $u$ is a strong solution of (1.1). Following [34], we consider its complex gradient $f=\partial u / \partial z$ as a mapping from $\Omega$ into $\mathbb{C}$. Then $f$ is a $K$ quasiregular mapping, which means that $f \in W_{\mathrm{loc}}^{1,2}(\Omega ; \mathbb{C})$ and

$$
\left|\frac{\partial f}{\partial \bar{z}}\right| \leq k\left|\frac{\partial f}{\partial z}\right|, \quad k=\frac{K-1}{K+1},
$$

a.e in $\Omega$. See, e.g. [5, 34, 49]. By Morrey's theorem [34] $f \in C_{\text {loc }}^{0,1 / K}(\Omega)$. The exponent $1 / K$ is best possible in the class of all $K$-quasiregular mapping, since this class contains the mapping $z \mapsto|z|^{1 / K-1} z$.

However, our $f$ has an additional property of being the complex gradient of a real-valued function. In particular, $\partial f / \partial \bar{z}$ is real-valued, because

$$
\frac{\partial f}{\partial \bar{z}}=\frac{\partial^{2} u}{\partial \bar{z} \partial z}=\frac{1}{4} \Delta u
$$

a.e. in $\Omega$. We call $f \in W_{\text {loc }}^{1,2}(\Omega ; \mathbb{C})$ a $K$-quasiregular gradient mapping $(K \geq 1)$ if (1.4) holds and $\operatorname{Im} \partial f / \partial \bar{z}=0$ a.e. in $\Omega$. By the above, the complex gradient of every strong solution of (1.1) is a $K$-quasiregular gradient mapping.

A partial converse of the last statement is also true. Namely, if $f: \Omega \rightarrow \mathbb{C}$ is a $K$-quasiregular gradient mapping and $\Omega$ is simply connected, then there exists a real-valued function $u \in W_{\mathrm{loc}}^{2,2}(\Omega)$ (unique up to an additive constant) such that $\partial u / \partial z=f$. This can be derived from the Poincaré lemma as in [28]. If $\Omega$ is not 
simply connected, the potential $u$ might not exist globally. For instance, $f(z)=$ $i z^{-1}$ is a holomorphic (i.e. a 1-quasiregular gradient) mapping in the annulus $\{z$ : $\left.2^{-1}<|z|<2\right\}$. If $f=\partial u / \partial z$ for some real-valued function $u$, then

$$
-2 \pi=\operatorname{Re} \int_{|z|=1} f(z) d z=-\operatorname{Im} \int_{0}^{2 \pi} e^{i \varphi} f\left(e^{i \varphi}\right) d \varphi=\frac{1}{2} \int_{0}^{2 \pi} \frac{\partial u}{\partial \varphi} d \varphi=0,
$$

a contradiction. In any case, once the existence of $u$ is known, it can be shown to be a strong solution of an equation of the form (1.1) with the ellipticity condition (1.2). This result goes back to Pucci [41]. See also [4, 32].

In [28] it was proved that $K$-quasiregular gradient mappings belong to the little Hölder space $c_{\text {loc }}^{0,1 / K}(\Omega)$. In other words, for every compact set $E \subset \Omega$

$$
\lim _{\delta \rightarrow 0} \delta^{-1 / K} \omega(\delta, E)=0,
$$

where $\omega(\delta, E)$ stands for the modulus of continuity of $f$ on $E$. This leads one to expect that strong solutions of (1.1) are locally $C^{1, \alpha}$ for some $\alpha>1 / K$. Our Theorem 1.1 confirms that this is indeed the case.

For divergence form equations the precise degree of Hölder regularity has been established by Piccinini and Spagnolo [40] (see also [21, 35, 44, 50]). It turns out that any weak solution of the equation

$$
\operatorname{div}(A(z) \nabla u(z))=0,
$$

with $A$ as in (1.2), is locally Hölder continuous with the exponent $1 / \sqrt{K}$. The sharpness of this result is demonstrated by the following example due to Meyers [33]. The function $u(z)=x|z|^{1 / \sqrt{K}-1}, z=x+i y$, is a weak solution of (1.5) with

$$
A(z)=\left(\begin{array}{cc}
1+(K-1) x^{2} /|z|^{2} & (K-1) x y /|z|^{2} \\
(K-1) x y /|z|^{2} & 1+(K-1) y^{2} /|z|^{2}
\end{array}\right) .
$$

This "pathological" differential equation is often associated (e.g. in [7] and [21]) with the name of Serrin, who studied it in [48].

Gilbarg and Serrin [19] introduced a certain class of non-divergence form operators which includes (1.6). Since then, such operators have become a frequent source of counterexamples [29, 31, 36, 42, 47]. In view of the Piccinini-Spagnolo theorem one might conjecture that the Gilbarg-Serrin operator with matrix (1.6) is the most "pathological" with a given ellipticity constant $K$ (that is, it exhibits the lowest degree of regularity of solutions).

D'Onofrio and Greco [15] recently established the best possible $C^{1, \alpha}$-regularity result for this equation in all dimensions $n \geq 2$. In particular, they proved that every strong solution of (1.1) with the coefficient matrix (1.6) is in $C_{\text {loc }}^{1, \alpha}$, where

$$
\alpha=\alpha_{K}^{(1)}:=\frac{1}{2}\left(\sqrt{1+14 K^{-1}+K^{-2}}-1-K^{-1}\right)
$$

cannot be replaced by any larger value. 
However, in Section 4 we construct a non-divergence form equation which is not of the Gilbarg-Serrin type and whose solutions are only $C_{\text {loc }}^{1, \alpha_{K}}$ with $\alpha_{K}<\alpha_{K}^{(1)}$. Moreover, Theorem 3.1 shows that $\alpha_{K}$ is the optimal exponent for all separated solutions of (1.1) (in particular, for all homogeneous solutions). It seems possible that the optimal exponent in the general case should be the same as in Theorem 3.1. So far, the best Hölder estimate we can prove for general solutions of (1.1) is the following.

Theorem 1.1. Suppose that $u \in W_{\mathrm{loc}}^{2,2}(\Omega)$ is a strong solution of (1.1) in a domain $\Omega \subset \mathbb{R}^{2}$, and that (1.2) holds. Then $u \in C_{\mathrm{loc}}^{1, \alpha}(\Omega)$, where

$$
\alpha=\alpha_{K}^{(2)}=\frac{1}{2(K+1)}\left(\sqrt{33+30 K^{-1}+K^{-2}}-3-K^{-1}\right) .
$$

One can show that

$$
\frac{1}{K}<\alpha_{K}^{(2)}<\alpha_{K}<\alpha_{K}^{(1)}<\min \left(\frac{1}{\sqrt{K}}, \frac{3}{K}\right)
$$

for all $K>1$. Furthermore,

$$
\begin{aligned}
& \lim _{K \rightarrow \infty} K \alpha_{K}^{(2)}=\frac{\sqrt{33}-3}{2} \approx 1.37 \\
& \lim _{K \rightarrow \infty} K \alpha_{K}=\lim _{K \rightarrow \infty} K \alpha_{K}^{(1)}=3 .
\end{aligned}
$$

Although Theorem 1.1 does not provide optimal Hölder exponents, it does provide the first Hölder regularity result for equation (1.1) beyond the long-standing threshold $1 / K$.

ACKnowledgements. We would like to thank Luigi D’Onofrio, Tadeusz Iwaniec and Carlo Sbordone for valuable discussions.

\section{Proof of Theorem 1.1}

We start by deriving a lower estimate for the Jacobian determinant of a quasiregular gradient mapping. Recall that we defined $f \in W_{\text {loc }}^{1,2}(\Omega ; \mathbb{C})$ to be a $K$-quasiregular gradient mapping if

$$
\operatorname{Im} \frac{\partial f}{\partial \bar{z}}=0 \text { and }\left|\frac{\partial f}{\partial \bar{z}}\right| \leq k\left|\frac{\partial f}{\partial z}\right|, \quad \text { where } k=\frac{K-1}{K+1},
$$

a.e. in $\Omega \subset \mathbb{C}$. The quasiregularity assumption (1.4) alone implies that $f$ has a non-negative Jacobian. In fact, if one writes $D f$ for the real derivative matrix of $f$ (i.e. $D f \in \mathbb{R}^{2 \times 2}$ ), then its determinant $J_{f}=\operatorname{det} D f$ satisfies

$$
J_{f} \geq K^{-1}|D f|^{2}, \quad \text { a.e. in } \Omega,
$$


where $|D f|$ is the operator norm of $D f$. Inequality (2.2) directly follows from (1.4) and the elementary identities

$$
\begin{aligned}
& |D f|=\left|\frac{\partial f}{\partial z}\right|+\left|\frac{\partial f}{\partial \bar{z}}\right| ; \\
& J_{f}(z)=\left|\frac{\partial f}{\partial z}\right|^{2}-\left|\frac{\partial f}{\partial \bar{z}}\right|^{2} .
\end{aligned}
$$

We shall replace (2.2) with a different estimate for which the full strength of the assumption (2.1) can be exploited. Since quasiregular mappings are differentiable a.e. $[43,45]$, we can use partial derivatives of $f$ in the polar coordinates $r, \varphi(1.3)$ to define real-valued functions $p, q \in L_{\text {loc }}^{2}(\Omega)$ such that

$$
i e^{i \varphi} r^{-1} \frac{\partial f}{\partial \varphi}(z)=p(z)+i q(z), \quad z=r e^{i \varphi},
$$

for a.e. $z \in \Omega$. Since $p^{2}+q^{2}=r^{-2}|\partial f / \partial \varphi|^{2} \leq|D f|^{2}$, it follows from (2.2) that

$$
J_{f} \geq K^{-1}\left(p^{2}+q^{2}\right) \text {. }
$$

The latter inequality is sharp in the class of $K$-quasiregular mappings, but it can be substantially improved in the subclass formed by gradients.

Lemma 2.1. Let $f: \Omega \rightarrow \mathbb{C}$ be a $K$-quasiregular gradient mapping. Then for a.e. $z \in \Omega$

$$
J_{f}(z) \geq K^{-1} p(z)^{2}+\frac{2}{K+1} q(z)^{2}=\frac{1-k}{1+k} p(z)^{2}+(1-k) q(z)^{2} .
$$

Proof. Since $\partial f / \partial \bar{z}$ is real-valued, so is the function

$$
s(z)=2 \frac{\partial f}{\partial \bar{z}}(z)-p(z)=e^{i \varphi} \frac{\partial f}{\partial r}(z)+i q(z), \quad z=r e^{i \varphi} .
$$

Note that

$$
\frac{\partial f}{\partial z}=\frac{e^{-2 i \varphi}}{2}(s-p-2 i q) \quad \text { and } \quad \frac{\partial f}{\partial \bar{z}}=\frac{1}{2}(s+p) .
$$

We can estimate $s(z)$ using (1.4):

$$
(s(z)+p(z))^{2} \leq k^{2}\left((s(z)-p(z))^{2}+4 q(z)^{2}\right),
$$

from which it follows by expanding squares and rearranging terms, that

$$
\left|s(z)+\frac{1+k^{2}}{1-k^{2}} p(z)\right| \leq \frac{2 k}{1-k^{2}} \sqrt{p(z)^{2}+\left(1-k^{2}\right) q(z)^{2}} .
$$


This implies the following estimate for the Jacobian determinant of $f$.

$$
\begin{aligned}
J_{f} & =\left|\frac{\partial f}{\partial z}\right|^{2}-\left|\frac{\partial f}{\partial \bar{z}}\right|^{2}=-\operatorname{Re}\left(\overline{\frac{\partial f}{\partial r}} \frac{i}{r} \frac{\partial f}{\partial \varphi}\right) \\
& =-\operatorname{Re}\left(e^{i \varphi \frac{\partial f}{\partial r}} \frac{e^{i \varphi}}{r} \frac{\partial f}{\partial \varphi}\right)=-\operatorname{Re}((s+i q)(p+i q))=q^{2}-p s \\
& \geq \frac{1+k^{2}}{1-k^{2}} p^{2}+q^{2}-\frac{2 k|p|}{1-k^{2}} \sqrt{p^{2}+\left(1-k^{2}\right) q^{2}} .
\end{aligned}
$$

For the purpose of integrating the Jacobian it is preferable to have an estimate that is linear in $p^{2}$ and $q^{2}$. Using the arithmetic-geometric mean inequality, we obtain

$$
2|p| \sqrt{p^{2}+\left(1-k^{2}\right) q^{2}} \leq 2 p^{2}+\left(1-k^{2}\right) q^{2} .
$$

From this and (2.4) the desired estimate (2.3) follows immediately.

Since Lemma 2.1 is a key to subsequent integral Jacobian estimates, it is natural to inquire about its sharpness. Theorem 4.1 gives an example of a $K$ quasiregular gradient mapping for which (2.4) turns into an equality for a.e. $\varphi \in$ $[0,2 \pi]$. The same cannot be said about (2.3), however. One could try to improve (2.3) by using a modified arithmetic-geometric mean inequality $2 a b \leq \mu a^{2}+$ $\mu^{-1} b^{2}, \mu>0$. Since this leads only to a very small improvement in the Hölder exponent (1.8), we do not pursue this matter here.

Proof of Theorem 1.1. First, let us introduce the notation $D\left(z_{0}, r\right)=\{z \in \mathbb{C}$ : $\left.\left|z-z_{0}\right|<r\right\}$. Also, fix $K>1$ and let $\alpha=\alpha_{K}^{(2)}$.

Suppose that $u$ is as in the statement of the theorem; then $f=\partial u / \partial z$ is a $K$ quasiregular gradient mapping from $\Omega$ to $\mathbb{C}$. Let $z_{0} \in \Omega$ and $0<R<\operatorname{dist}\left(z_{0}, \partial \Omega\right)$. Our goal is to prove that

$$
\int_{D\left(z_{0}, r\right)}|D f(z)|^{2} d \mathcal{L}^{2}(z) \leq K\left(\frac{r}{R}\right)^{2 \alpha} \int_{D\left(z_{0}, R\right)}|D f(z)|^{2} d \mathcal{L}^{2}(z), \quad 0 \leq r \leq R
$$

where $\mathcal{L}^{2}$ is the 2-dimensional Lebesgue measure. Once we have (2.5), Morrey's lemma $[20,12.2]$ will imply $f \in C_{\mathrm{loc}}^{0, \alpha}(\Omega)$, i.e. the conclusion of the theorem.

Without loss of generality we may assume that $z_{0}=0$ and $R=1$. Recall that $K^{-1}|D f|^{2} \leq J_{f} \leq|D f|^{2}$ by virtue of Hadamard's determinant inequality and (2.2). Thus the estimate (2.5) will follow once we prove

$$
\int_{D(0, r)} J_{f}(z) d \mathcal{L}^{2}(z) \leq r^{2 \alpha} \int_{D(0,1)} J_{f}(z) d \mathcal{L}^{2}(z), \quad 0 \leq r \leq 1
$$


Let $J(r)$ denote the left-hand side of (2.6). It is evident that $J$ is an increasing absolutely continuous function on the interval [0,1]. Following Morrey [34], we are going to estimate its derivative $J^{\prime}(r)$ from below for almost all $r \in(0,1)$.

For a.e. $r \in(0,1)$ the function $\varphi \mapsto f\left(r e^{i \varphi}\right)$ is absolutely continuous and its derivative is square integrable. This allows us to expand it into the uniformly converging Fourier series

$$
f\left(r e^{i \varphi}\right)=\sum_{n \in \mathbb{Z}} c_{n}(r) e^{i n \varphi},
$$

where

$$
c_{n}(r)=\frac{1}{2 \pi} \int_{0}^{2 \pi} f\left(r e^{i \varphi}\right) e^{-i n \varphi} d \varphi, \quad n \in \mathbb{Z} .
$$

Furthermore, the series (2.7) can be differentiated term by term [51, II.2.1] to obtain

$$
\frac{\partial f\left(r e^{i \varphi}\right)}{\partial \varphi}=\sum_{n \in \mathbb{Z}} i n c_{n}(r) e^{i n \varphi},
$$

with the latter series converging in $L^{2}[0,2 \pi]$. Let $r \in(0,1)$ be such that for a.e. $\varphi \in[0,2 \pi]$ the function $f$ is differentiable at $r e^{i \varphi}$ and (2.1) holds. Using Green's formula and Parseval's theorem, we find

$$
J(r)=\frac{1}{2 i} \int_{0}^{2 \pi} \bar{f}\left(r e^{i \varphi}\right) \frac{\partial f}{\partial \varphi}\left(r e^{i \varphi}\right) d \varphi=\pi \sum_{n \in \mathbb{Z}} n\left|c_{n}(r)\right|^{2} .
$$

Let $d_{n}=n c_{n}(r)$. Since

$$
p\left(r e^{i \varphi}\right)+i q\left(r e^{i \varphi}\right)=\frac{e^{i \varphi} i}{r} \sum_{n \in \mathbb{Z}} i n c_{n}(r) e^{i n \varphi}=-\frac{1}{r} \sum_{n \in \mathbb{Z}} d_{n-1} e^{i n \varphi},
$$

it follows that

$$
\begin{aligned}
\int_{0}^{2 \pi} p\left(r e^{i \varphi}\right)^{2} d \varphi & =\frac{2 \pi}{r^{2}} \sum_{n \in \mathbb{Z}}\left|\left(d_{n-1}+\bar{d}_{-n-1}\right) / 2\right|^{2} \\
& =\frac{\pi}{2 r^{2}} \sum_{n \in \mathbb{Z}}\left|d_{n-1}+\bar{d}_{-n-1}\right|^{2}
\end{aligned}
$$

and

$$
\begin{aligned}
\int_{0}^{2 \pi} q\left(r e^{i \varphi}\right)^{2} d \varphi & =\frac{2 \pi}{r^{2}} \sum_{n \in \mathbb{Z}}\left|\left(d_{n-1}-\bar{d}_{-n-1}\right) / 2\right|^{2} \\
& =\frac{\pi}{2 r^{2}} \sum_{n \in \mathbb{Z}}\left|d_{n-1}-\bar{d}_{-n-1}\right|^{2}
\end{aligned}
$$


Now integrate $(2.3)$ over $\varphi \in[0,2 \pi]$ to obtain

$$
\begin{aligned}
J^{\prime}(r) & =r \int_{0}^{2 \pi} J_{f}\left(r e^{i \varphi}\right) d \varphi \\
& \geq \frac{1-k}{1+k} r \int_{0}^{2 \pi}\left(p\left(r e^{i \varphi}\right)^{2}+(1+k) q\left(r e^{i \varphi}\right)^{2}\right) d \varphi \\
& =\frac{\pi(1-k)}{2 r(1+k)} \sum_{n \in \mathbb{Z}}\left\{\left|d_{n-1}+\bar{d}_{-n-1}\right|^{2}+(1+k)\left|d_{n-1}-\bar{d}_{-n-1}\right|^{2}\right\} \\
& \geq \frac{\pi(1-k)}{r(1+k)} \sum_{n=2}^{\infty}\left\{\left|d_{n-1}+\bar{d}_{-n-1}\right|^{2}+(1+k)\left|d_{n-1}-\bar{d}_{-n-1}\right|^{2}\right\}
\end{aligned}
$$

On the other hand, (2.8) implies

$$
J(r)=\pi \sum_{n \in \mathbb{Z}} n\left|c_{n}(r)\right|^{2}=\pi \sum_{n \neq 0} \frac{\left|d_{n}\right|^{2}}{n} \leq \pi \sum_{n=2}^{\infty}\left\{\frac{\left|d_{n-1}\right|^{2}}{n-1}-\frac{\left|d_{-n-1}\right|^{2}}{n+1}\right\} .
$$

In order to compare $J^{\prime}(r)$ and $J(r)$, we must find the largest possible constant $B$ such that the inequality

$$
\left|d_{n-1}+\bar{d}_{-n-1}\right|^{2}+(1+k)\left|d_{n-1}-\bar{d}_{-n-1}\right|^{2} \geq B\left\{\frac{\left|d_{n-1}\right|^{2}}{n-1}-\frac{\left|d_{-n-1}\right|^{2}}{n+1}\right\}
$$

holds for all $n \geq 2$. Because of homogeneity of (2.13) it suffices to consider the case $d_{n-1}=1, \bar{d}_{-n-1}=\zeta \in \mathbb{C}$. Furthermore, if $\zeta$ is replaced with $\operatorname{Re} \zeta$, the left-hand side of (2.13) decreases while the other side increases. So (2.13) reduces to

$$
(1+\zeta)^{2}+(1+k)(1-\zeta)^{2} \geq B\left\{\frac{1}{n-1}-\frac{\zeta^{2}}{n+1}\right\}, \quad \zeta \in \mathbb{R}
$$

Since the left-hand side of (2.14) is at least 2 , we can take $B=4$ when $n \geq 3$. Let us consider the case $n=2$ in more detail. The quadratic polynomial

$$
(1+\zeta)^{2}+(1+k)(1-\zeta)^{2}-B\left(1-\zeta^{2} / 3\right)=(2+k+B / 3) \zeta^{2}-2 k \zeta+(2+k-B)
$$

is non-negative for all $\zeta \in \mathbb{R}$ if and only if its discriminant is non-positive, that is,

$$
k^{2} \leq(2+k+B / 3)(2+k-B) .
$$

The largest value of $B$ that verifies the latter inequality is

$$
B=\sqrt{k^{2}+16 k+16}-k-2 .
$$


Since $\sqrt{k^{2}+16 k+16}-k-2<\sqrt{33}-2<4$, the value of $B$ given by (2.15) applies to all $n \geq 2$.

Combining (2.11), (2.12) and (2.13), we obtain

$$
J^{\prime}(r) \geq B \frac{1-k}{1+k} \frac{J(r)}{r}, \quad \text { a.e. } r \in(0,1) .
$$

Using (1.8) and the identity $k=(K-1) /(K+1)$, it is straightforward to verify that

$$
\left(\sqrt{k^{2}+16 k+16}-k-2\right) \frac{1-k}{1+k}=2 \alpha .
$$

It remains to integrate the differential inequality (2.16) and obtain

$$
J(r) \leq r^{2 \alpha} J(1)
$$

This completes the proof of (2.6) and of Theorem 1.1.

\section{The best possible Hölder exponent for separated solutions}

Given a number $K>1$, let $k=(K-1) /(K+1)$ and define $k_{0} \in(0,1)$ by the following equation.

$$
\frac{k}{\sqrt{1-k^{2}}} \tan ^{-1} \frac{k}{\sqrt{1-k^{2}}}=\frac{k_{0}}{\sqrt{1-k_{0}^{2}}}\left(\frac{\pi}{2}+\tan ^{-1} \frac{k_{0}}{\sqrt{1-k_{0}^{2}}}\right) .
$$

Evidently $k_{0}<k$. Now define $\alpha_{K} \in(0,1)$ by

$$
\frac{1-\alpha_{K}}{1+\alpha_{K}} k=k_{0}, \quad \text { i.e. } \quad \alpha_{K}=\frac{k-k_{0}}{k+k_{0}} .
$$

The complicated definition of $\alpha_{K}$ is justified by the fact that it turns out to be the optimal Hölder exponent for at least a large class of solutions of (1.1).

Theorem 3.1. Let $\Omega=\mathbb{C}$. Suppose that $u$ is a strong solution of (1.1) in $\mathbb{C}$, and that (1.2) holds. Suppose in addition that $u$ has the form

$$
u\left(r e^{i \varphi}\right)=g(r) p(\varphi), \quad r \geq 0, \varphi \in \mathbb{R} .
$$

Then $u \in C_{\mathrm{loc}}^{1, \alpha_{K}}(\mathbb{C})$, where $\alpha_{K}$ is defined by (3.2). The exponent $\alpha_{K}$ is best possible.

An important special case of Theorem 3.1 is the case when $u$ is homogeneous, i.e. $u\left(r e^{i \varphi}\right)=r^{s} p(\varphi)$.

The proof of Theorem 3.1 will be carried out in several steps, the first of which is to reduce the problem to a special case. To avoid trivialities, assume that $u$ is 
nonconstant. Then $p$ must be nonconstant as well, for otherwise either $u$ or $-u$ would violate the maximum principle [20, 9.6]. Continuity of $u$ forces $g(0)=0$. The maximum principle then implies that $g(r) \neq 0$ for $r>0$; we may assume $g(r)>0$. Since $u$ is continuously differentiable, so are the functions $g$ and $p$. Next we reduce the problem to the case $g^{\prime}(0)=0$. The first-order Taylor expansion of $u$ at 0 can be written as

$$
u\left(r e^{i \varphi}\right)=g(r) p(\varphi)=\operatorname{Re}\left(\bar{a} r e^{i \varphi}\right)+o(r),
$$

where $a \in \mathbb{C}$. If $a \neq 0$, then after dividing by $r$ and passing to the limit $r \rightarrow 0$ we obtain

$$
p(\varphi)=|a| g^{\prime}(0)^{-1} \cos (\varphi+\arg a) .
$$

Since the function $r e^{i \varphi} \mapsto g^{\prime}(0) r p(\varphi)$ is linear, the difference

$$
u_{1}\left(r e^{i \varphi}\right)=u\left(r e^{i \varphi}\right)-g^{\prime}(0) r p(\varphi)=\left(g(r)-g^{\prime}(0) r\right) p(\varphi)
$$

is a solution of (1.1) with the same degree of regularity as $u$. Moreover, $u_{1}$ has the form $u_{1}\left(r e^{i \varphi}\right)=\widetilde{g}(r) p(\varphi)$ with $\widetilde{g}^{\prime}(0)=0$.

The assumptions

$$
g(0)=g^{\prime}(0)=0, \quad u \not \equiv 0, \quad \text { and } \quad g(r)>0 \text { for } r>0,
$$

as well as the hypotheses of Theorem 3.1, are in force for the rest of the section.

\section{Lemma 3.2.}

(i) $u \in C_{\mathrm{loc}}^{1,1}(\mathbb{C} \backslash\{0\})$;

(ii) the function $\beta(r):=r g^{\prime}(r) / g(r)$ is locally absolutely continuous on $(0, \infty)$;

(iii) there exist $r_{0}>0$ and $H<\infty$ such that

$$
1<\beta(r)<H, \quad 0<r<r_{0} .
$$

Proof. Using the absolute continuity of Sobolev functions on lines [16, 4.9.2] in the context of polar coordinates, we find that $p \in W_{\mathrm{loc}}^{2,2}(\mathbb{R})$ and $g \in W_{\mathrm{loc}}^{2,2}(0, \infty)$. Let $f=\partial u / \partial z$. Then, by (1.3),

$$
f\left(r e^{i \varphi}\right)=\frac{e^{-i \varphi}}{2}\left(g^{\prime}(r) p(\varphi)-\frac{i}{r} g(r) p^{\prime}(\varphi)\right) .
$$

Choose $r_{1}>0$ so that $g^{\prime \prime}\left(r_{1}\right)$ exists. Differentiating (3.4) in $r$ we obtain

$$
\limsup _{r \rightarrow r_{1}} \frac{\left|f\left(r e^{i \varphi}\right)-f\left(r_{1} e^{i \varphi}\right)\right|}{\left|r-r_{1}\right|} \leq C, \quad \varphi \in \mathbb{R},
$$

with $C$ finite and independent of $\varphi$. Quasiregularity of $f$ now implies [45, II.4.3]

$$
\limsup _{\varphi \rightarrow \varphi_{1}} \frac{\left|f\left(r_{1} e^{i \varphi}\right)-f\left(r_{1} e^{i \varphi_{1}}\right)\right|}{r_{1}\left|\varphi-\varphi_{1}\right|} \leq C_{1}, \quad \varphi_{1} \in \mathbb{R},
$$


where $C_{1}$ does not depend on $\varphi_{1}$. Comparing (3.5) with (3.4), we see that $p^{\prime}$ is a Lipschitz function on $\mathbb{R}$. Hence the restriction of $f$ to every circle centered at the origin is Lipschitz. Invoking Theorem II.4.3 [45] again, we conclude that $f$ is locally Lipschitz in $\mathbb{C} \backslash\{0\}$. This proves (i).

The strong maximum principle [20,9.6] implies that $g^{\prime}(r) \geq 0$ for all $r>0$. Thus the function $\beta(r):=r g^{\prime}(r) / g(r)$ is continuous and nonnegative for $r>0$. Since $g^{\prime}$ is absolutely continuous on every interval $\left[r_{1}, r_{2}\right]$ with $0<r_{1}<r_{2}<\infty$, part (ii) follows.

Next we want to prove that $g^{\prime}(r)$ is strictly increasing, at least for small values of $r$. Since the branch set of the quasiregular mapping $f=\partial u / \partial z$ is discrete [45], there exists $r_{0}>0$ such that $f$ is a local homeomorphism in $D\left(0, r_{0}\right) \backslash\{0\}$. Fix $\varphi_{1} \in \mathbb{R}$ such that $p\left(\varphi_{1}\right)=\max _{\varphi \in \mathbb{R}} p(\varphi)$. Since

$$
f\left(r e^{i \varphi_{1}}\right)=\frac{e^{-i \varphi_{1}}}{2} g^{\prime}(r) p\left(\varphi_{1}\right), \quad r>0,
$$

$f$ maps the halfline $\left\{r e^{i \varphi_{1}}: r>0\right\}$ into another halfline $\left\{r e^{-i \varphi_{1}}: r>0\right\}$. The homeomorphism property of $f$ implies that $\operatorname{Re} e^{i \varphi_{1}} f\left(r e^{i \varphi_{1}}\right)$ strictly increases with $r$ when $0<r<r_{0}$. Hence $g^{\prime}(r)$ is a strictly increasing function for $0<r<r_{0}$. From $g(r)=\int_{0}^{r} g^{\prime}$, we easily obtain $\beta(r)>1,0<r<r_{0}$.

Since $u(0)=0$, by the maximum principle there exists $\varphi_{2} \in \mathbb{R}$ such that $p\left(\varphi_{2}\right)=0$. We have

$$
f\left(r e^{i \varphi_{2}}\right)=-\frac{i e^{-i \varphi_{2}} g(r)}{2 r} p^{\prime}\left(\varphi_{2}\right), \quad r>0 .
$$

Since $f(0)=0$, Theorem II.4.3 [45] implies that the ratio

$$
\frac{\max _{\varphi}\left|f\left(r e^{i \varphi}\right)\right|}{\min _{\varphi}\left|f\left(r e^{i \varphi}\right)\right|}
$$

is uniformly bounded for all sufficiently small $r>0$. Combining this with (3.6) and (3.7), we obtain (iii).

Before stating the next lemma, recall that $k=(K-1) /(K+1)$ and introduce an auxiliary function of four real variables

$$
F(x, y, s, t)=\left(x+y^{2}+t\right)^{2}-k^{2}\left\{\left(x+y^{2}+2 s-t\right)^{2}+4(s-1)^{2} y^{2}\right\} .
$$

Let us call a positive number $r$ a regular radius if at a.e. point of the circle $\{z:|z|=$ $r$ \} the mapping $f$ is differentiable and (1.4) holds. By Fubini's theorem a.e. $r>0$ is a regular radius.

Lemma 3.3. There exists an interval $(a, b) \subset \mathbb{R}$ such that $b-a \leq \pi / 2, p(a)=$ $p(b)=0$, and $p(\varphi) \neq 0$ for $\varphi \in(a, b)$. Define $q(\varphi)=p^{\prime}(\varphi) / p(\varphi), \varphi \in(a, b)$. Then for every regular radius $r>0$ we have

$$
F\left(q^{\prime}(\varphi), q(\varphi), \beta(r), \beta(r)^{2}+r \beta^{\prime}(r)\right) \leq 0
$$

for a.e. $\varphi \in(a, b)$. 
Proof. Since $f^{-1}(0)$ is a discrete set [43, 45], there exists $r>0$ such that the mapping $f=\partial u / \partial z$ does not vanish on the circle $\{z \in \mathbb{C}:|z|=r\}$. Since $f$ is orientation-preserving and $f(0)=0$, it follows that the closed curve $\varphi \mapsto f\left(r e^{i \varphi}\right)$, $\varphi \in[0,2 \pi]$, has index 1 or more with respect to the origin. Therefore, the closed curve $\varphi \mapsto e^{i \varphi} f\left(r e^{i \varphi}\right), \varphi \in[0,2 \pi]$, has index 2 or more with respect to the origin. In particular, it intersects the imaginary axis at least 4 times. Since

$$
\operatorname{Re}\left(e^{i \varphi} f\left(r e^{i \varphi}\right)\right)=\frac{1}{2} \frac{\partial u}{\partial r}=\frac{1}{2} g^{\prime}(r) p(\varphi),
$$

the function $p$ has at least 4 zeroes on every closed interval of length $2 \pi$. This implies the existence of $(a, b) \subset \mathbb{R}$ as in the statement of the lemma.

Now let us fix a regular radius $r>0$, write $\beta=\beta(r)$, and set

$$
\zeta=\beta(r)^{2}+r \beta^{\prime}(r)=\frac{r g^{\prime}(r)}{g(r)}+\frac{r^{2} g^{\prime \prime}(r)}{g(r)},
$$

where $\beta(r)$ is as in Lemma 3.2. At a.e. point of $\{z:|z|=r\}$ we have

$$
\begin{aligned}
4 \frac{\partial f}{\partial \bar{z}} & =\Delta u=\frac{\partial^{2} u}{\partial r^{2}}+\frac{1}{r} \frac{\partial u}{\partial r}+\frac{1}{r^{2}} \frac{\partial^{2} u}{\partial \varphi^{2}}=\frac{g(r)}{r^{2}}\left(p^{\prime \prime}+\zeta p\right) ; \\
-4 e^{2 i \varphi} \frac{\partial f}{\partial z} & =-\frac{\partial^{2} u}{\partial r^{2}}+\frac{1}{r} \frac{\partial u}{\partial r}+\frac{1}{r^{2}} \frac{\partial^{2} u}{\partial \varphi^{2}}+2 i\left(\frac{1}{r} \frac{\partial^{2} u}{\partial r \partial \varphi}-\frac{1}{r^{2}} \frac{\partial u}{\partial \varphi}\right) \\
& =\frac{g(r)}{r^{2}}\left(p^{\prime \prime}+(2 \beta-\zeta) p+2 i(\beta-1) p^{\prime}\right) .
\end{aligned}
$$

Inequality (1.4) can now be rewritten as an ordinary differential inequality that holds a.e. on $(a, b)$.

$$
\left(p^{\prime \prime}+\zeta p\right)^{2} \leq k^{2}\left\{\left(p^{\prime \prime}+(2 \beta-\zeta) p\right)^{2}+4(\beta-1)^{2}\left(p^{\prime}\right)^{2}\right\} .
$$

Dividing (3.10) by $p^{2}$ and using the identity $p^{\prime \prime}=q^{\prime}+q^{2}$, we obtain (3.8).

Let

$$
E=\left\{(s, t) \in \mathbb{R}^{2}: F\left(q^{\prime}(\varphi), q(\varphi), s, t\right) \leq 0 \text { for a.e. } \varphi \in(a, b)\right\} ;
$$

Lemma 3.3 says that $\left(\beta(r), \beta(r)^{2}+r \beta^{\prime}(r)\right) \in E$ for every regular radius $r$. Furthermore, the definition of $F$ implies that $E$ is a closed set that is convex in the $t$-direction. The latter means that if $\left(s, t_{j}\right) \in E, j=1,2$, then $(s, t) \in E$ for all values of $t$ between $t_{1}$ and $t_{2}$.

Lemma 3.4. In the notation of Lemma 3.3, $q^{\prime}(\varphi)<0$ for a.e. $\varphi \in(a, b)$. 
Proof. Let $s_{0}=\liminf _{r \rightarrow 0} \beta(r)$; by (3.3) $s_{0} \geq 1$. We claim that $\left(s_{0}, s_{0}^{2}\right) \in E$. If $\beta(r)=s_{0}$ for all $r>0$, then the claim follows from Lemma 3.3. Suppose that $\beta$ is nonconstant. Introduce the sets

$$
B=\{\beta(r): r>0 \text { is not a regular radius }\} \text { and } G=\{\beta(r): r>0\} \backslash B .
$$

Notice that $B$ is the image under $\beta$ of a set of measure zero. Since $\beta$ is a locally absolutely continuous function, $B$ has measure zero as well. Let us consider two possibilities.

Case 1. The function $\beta$ is monotone in some right neighborhood of 0 . Then $\lim _{r \rightarrow 0} \beta(r)=s_{0}$. Also, the bound (3.3) then implies ess $\liminf _{r \rightarrow 0} r\left|\beta^{\prime}(r)\right|=0$. Therefore, we can find a sequence of regular radii $\left\{r_{j}\right\}$ such that $\beta\left(r_{j}\right) \rightarrow s_{0}$ and $r_{j} \beta^{\prime}\left(r_{j}\right) \rightarrow 0$. Since $E$ is a closed set, we have $\left(s_{0}, s_{0}^{2}\right) \in E$.

Case 2. The function $\beta$ is not monotone in any neighborhood of 0 . Then we can find a sequence $\left\{s_{j}\right\} \subset G$ such that $s_{j} \rightarrow s_{0}$ and for every $j$ the set $\beta^{-1}\left(s_{j}\right)$ contains more than one point. Given such $j$, there exist $r_{1}, r_{2} \in \beta^{-1}\left(s_{j}\right)$ such that $\beta^{\prime}\left(r_{1}\right) \leq 0 \leq \beta^{\prime}\left(r_{2}\right)$. Since $\left(s_{j}, s_{j}^{2}+r_{l} \beta^{\prime}\left(r_{l}\right)\right) \in E$ for $l=1,2$, the partial convexity of $E$ implies $\left(s_{j}, s_{j}^{2}\right) \in E$. Finally, $\left(s_{0}, s_{0}^{2}\right) \in E$ because $E$ is closed.

So far we have proved that $\left(s_{0}, s_{0}^{2}\right) \in E$, which means

$$
F\left(q^{\prime}(\varphi), q(\varphi), s_{0}, s_{0}^{2}\right) \leq 0, \quad \text { a.e. } \varphi \in(a, b) .
$$

Observe that $F(x, y, s, t)$ is a quadratic polynomial in $x$ with a positive leading coefficient. Hence for any $y, s, t \in \mathbb{R}$ the set $\{x \in \mathbb{R}: F(x, y, s, t) \leq 0\}$ is a subinterval of $\mathbb{R}$. It is easy to check that for every $y \in \mathbb{R}$

$$
F\left(-y^{2}-s_{0}^{2}, y, s_{0}, s_{0}^{2}\right)=-k^{2}\left\{\left(2 s_{0}-2 s_{0}^{2}\right)^{2}+4\left(s_{0}-1\right)^{2} y^{2}\right\}<0
$$

and

$$
F\left(0, y, s_{0}, s_{0}^{2}\right)=\left(y^{2}+s_{0}^{2}\right)\left(\left(1-k^{2}\right)\left(y^{2}+s_{0}^{2}\right)+4 k^{2}\left(s_{0}-1\right)\right)>0 .
$$

Therefore, $\left\{x \in \mathbb{R}: F\left(x, y, s_{0}, s_{0}^{2}\right) \leq 0\right\} \subset(-\infty, 0)$. This and (3.11) imply the conclusion of the lemma.

The following computations lie at the core of our proof of Theorem 3.1.

Lemma 3.5. Let $q$ and $E$ be as above. If $(s, t) \in E$ is such that $s>1$ and $t \leq s^{2}$, then $s \geq 1+\alpha_{K}$.

Proof. Solving the inequality $F\left(q^{\prime}, q, s, t\right) \leq 0$ for $q^{\prime}$, we obtain

$$
\left|q^{\prime}+q^{2}+\frac{\left(1+k^{2}\right) t-2 k^{2} s}{1-k^{2}}\right| \leq \frac{2 k}{1-k^{2}} \sqrt{(t-s)^{2}+\left(1-k^{2}\right)(s-1)^{2} q^{2}} .
$$


Let

$$
\begin{aligned}
R(x, y, s, t)= & x+y^{2}+\frac{\left(1+k^{2}\right) t-2 k^{2} s}{1-k^{2}} \\
& +\frac{2 k}{1-k^{2}} \sqrt{(t-s)^{2}+\left(1-k^{2}\right)(s-1)^{2} y^{2}}
\end{aligned}
$$

inequality (3.12) implies that $R\left(q^{\prime}(\varphi), q(\varphi), s, t\right) \geq 0$ for a.e. $\varphi \in(a, b)$. We claim that $R$ is an increasing function of $t$. To verify this, observe that for any fixed $x, y$ and $s$ the function $R$ has the form

$$
t \mapsto c_{1}+c_{2} t+c_{3} \sqrt{(t-s)^{2}+c_{4}}
$$

where $c_{2}>c_{3}>0$ and $c_{4} \geq 0$. Differentiation yields

$$
\frac{\partial R}{\partial t}=c_{2}+c_{3} \frac{t-s}{\sqrt{(t-s)^{2}+c_{4}}} \geq c_{2}-c_{3}>0
$$

as required.

The conditions $p(a)=0=p(b)$ imply that for every $c \in(a, b)$

$$
\int_{a}^{c} q(\varphi) d \varphi=+\infty \text { and } \int_{c}^{b} q(\varphi) d \varphi=-\infty .
$$

Since $q$ is strictly decreasing by Lemma 3.4, it follows that

$$
\lim _{\varphi \rightarrow a} q(\varphi)=+\infty \quad \text { and } \quad \lim _{\varphi \rightarrow b} q(\varphi)=-\infty .
$$

Since $q(\varphi)$ is strictly decreasing, we can consider the inverse function $\varphi(q)$ which maps $\mathbb{R}$ onto the interval $(a, b)$. By the above monotonicity of $R$ we have $R\left(q^{\prime}, q, s, s^{2}\right) \geq R\left(q^{\prime}, q, s, t\right) \geq 0$. From the definition of $R\left(q^{\prime}, q, s, s^{2}\right)$ it follows that at a.e. point of $\mathbb{R}$

$$
\frac{d \varphi}{d q} \leq-\left\{q^{2}+\frac{2 k(s-1)}{1-k^{2}} \sqrt{\left(1-k^{2}\right) q^{2}+s^{2}}+\frac{s^{2}-k^{2} s(2-s)}{1-k^{2}}\right\}^{-1}
$$

Taking an antiderivative (with respect to $q$ ) of the right-hand side of (3.14), we obtain

$$
\begin{aligned}
& \Phi_{k, s}(q):=\frac{1}{2} \tan ^{-1}\left(\frac{k q}{\sqrt{\left(1-k^{2}\right) q^{2}+s^{2}}}\right)-\frac{1}{2} \tan ^{-1}\left(\frac{q}{s}\right) \\
& -\frac{k_{1} \sqrt{1-k^{2}}}{2 k \sqrt{1-k_{1}^{2}}}\left\{\tan ^{-1}\left(\frac{q \sqrt{1-k^{2}}}{s \sqrt{1-k_{1}^{2}}}\right)+\tan ^{-1}\left(\frac{k_{1}}{\sqrt{1-k_{1}^{2}}} \frac{q \sqrt{1-k^{2}}}{\sqrt{\left(1-k^{2}\right) q^{2}+s^{2}}}\right)\right\},
\end{aligned}
$$


where

$$
k_{1}=\frac{2-s}{s} k<k<1 .
$$

(It is straightforward, although a bit tiresome, to verify the antiderivative formula by differentiating $\Phi_{k, s}$ in $q$.) Now we integrate (3.14) over $\mathbb{R}$ :

$$
\begin{aligned}
b-a & =-\int_{-\infty}^{\infty} \frac{d \varphi}{d q} d q \geq \Phi_{k, s}(-\infty)-\Phi_{k, s}(\infty) \\
& =\frac{\pi}{2}-\tan ^{-1}\left(\frac{k}{\sqrt{1-k^{2}}}\right)+\frac{k_{1} \sqrt{1-k^{2}}}{k \sqrt{1-k_{1}^{2}}}\left\{\frac{\pi}{2}+\tan ^{-1}\left(\frac{k_{1}}{\sqrt{1-k_{1}^{2}}}\right)\right\} .
\end{aligned}
$$

Since $b-a \leq \pi / 2$, it follows that

$$
\frac{k}{\sqrt{1-k^{2}}} \tan ^{-1} \frac{k}{\sqrt{1-k^{2}}} \geq \frac{k_{1}}{\sqrt{1-k_{1}^{2}}}\left(\frac{\pi}{2}+\tan ^{-1} \frac{k_{1}}{\sqrt{1-k_{1}^{2}}}\right) .
$$

Comparing (3.16) with (3.1) we conclude that $k_{1} \leq k_{0}$. This together with (3.2) and (3.15) imply $s \geq 1+\alpha_{K}$.

Proof of Theorem 3.1. We claim that $\beta(r) \geq 1+\alpha_{K}$ for $0<r<r_{0}$, where $r_{0}$ is as in (3.3). Suppose, to the contrary, that there exists $\rho \in\left(0, r_{0}\right)$ such that $\beta(\rho)<1+\alpha_{K}$. Recall that $\beta$ is locally absolutely continuous on $\left(0, r_{0}\right)$ and that $\left(\beta(r), \beta(r)^{2}+r \beta^{\prime}(r)\right) \in E$ for every regular radius $r \in\left(0, r_{0}\right)$. By virtue of Lemma 3.5 we have $\beta^{\prime}(r)>0$ whenever $r \in\left(0, r_{0}\right)$ is a regular radius such that $\beta(r)<1+\alpha_{K}$. Therefore, $\beta^{\prime}(r)>0$ for every regular radius $r \in(0, \rho)$. Since $\beta$ is bounded from below by (3.3), it follows that ess $\liminf _{r \rightarrow 0} r \beta^{\prime}(r)=0$. Let $s_{0}=\lim _{r \rightarrow 0} \beta(r)$. Since $E$ is closed, we have $\left(s_{0}, s_{0}^{2}\right) \in E$. Using Lemma 3.5 again, we conclude that $s_{0} \geq 1+\alpha_{K}$. But $\beta(\rho)>s_{0}$ because $\beta$ is increasing on $(0, \rho)$. This is a contradiction.

Integrating the inequality $\beta(r) \geq 1+\alpha_{K}$ with respect to $r$, we obtain $g(r)=$ $O\left(r^{1+\alpha_{K}}\right), r \rightarrow 0$. Furthermore, (3.3) implies $g^{\prime}(r)=O\left(r^{\alpha_{K}}\right)$. By virtue of formula (3.4) and Lemma 3.2 (i) the gradient mapping $f$ is locally Hölder continuous in $\mathbb{C}$ with exponent $\alpha_{K}$.

The sharpness of Theorem 3.1 is demonstrated in the next section.

\section{Sharpness of Theorem 3.1}

Theorem 4.1. There exists a $K$-quasiregular gradient mapping $f: \mathbb{C} \rightarrow \mathbb{C}$ which is homogeneous of degree $\alpha_{K}$, with $\alpha_{K}$ as in (3.2). 
Proof. The construction of $f$ uses some elements of the proof of Lemma 3.5, in particular the function $\Phi_{k, s}$. Let $k$ and $k_{0}$ be as in the definition of $\alpha_{K}$ at the beginning of Section 3. Let $s=1+\alpha_{K}$ and observe that

$$
k_{1}=\frac{2-s}{s} k=\frac{1-\alpha_{K}}{1+\alpha_{K}} k=k_{0} .
$$

Now define a function $\varphi: \mathbb{R} \rightarrow(-\pi / 4, \pi / 4)$ by $\varphi(q)=\Phi_{k, s}(q)$. Using (3.1) we obtain

$$
\begin{aligned}
\lim _{q \rightarrow-\infty} \varphi(q) & =-\frac{1}{2} \tan ^{-1} \frac{k}{\sqrt{1-k^{2}}}+\frac{\pi}{4}+\frac{k_{0} \sqrt{1-k^{2}}}{2 k \sqrt{1-k_{0}^{2}}}\left\{\frac{\pi}{2}+\tan ^{-1} \frac{k_{0}}{\sqrt{1-k_{0}^{2}}}\right\} \\
& =\frac{\pi}{4}
\end{aligned}
$$

Also, $\lim _{q \rightarrow \infty} \varphi(q)=-\pi / 4$ because $\varphi(q)$ is an odd function. Since $\varphi^{\prime}(q)$ is the right-hand side of (3.14), we have

$$
\varphi^{\prime}(q)=-\left\{q^{2}+\frac{2 k(s-1)}{1-k^{2}} \sqrt{\left(1-k^{2}\right) q^{2}+s^{2}}+\frac{s^{2}-k^{2} s(2-s)}{1-k^{2}}\right\}^{-1}<0
$$

so there exists an inverse function $q:(-\pi / 4, \pi / 4) \rightarrow \mathbb{R}$. From (4.1) we obtain

$$
q^{\prime}+q^{2}+\frac{s^{2}-k^{2} s(2-s)}{1-k^{2}}=-\frac{2 k(s-1)}{1-k^{2}} \sqrt{\left(1-k^{2}\right) q^{2}+s^{2}} .
$$

Some algebra gives

$$
\left(q^{\prime}+q^{2}+s^{2}\right)^{2}=k^{2}\left\{\left(q^{\prime}+q^{2}+s(2-s)\right)^{2}+4(s-1)^{2} q^{2}\right\} .
$$

Now let

$$
p(\varphi)=\exp \left(\int_{0}^{\varphi} q(t) d t\right), \quad|\varphi|<\frac{\pi}{4}
$$

Obviously $p^{\prime} / p=q$; furthermore, $p$ is an even function. In order to continuously extend $p$ to the real line $\mathbb{R}$, we must first verify that $\lim _{\varphi \rightarrow \pi / 4} p(\varphi)=0$, i.e. $\int_{0}^{\pi / 4} q(t) d t=-\infty$. Changing the variable of integration to $q$, we obtain from (4.1)

$$
\int_{0}^{\pi / 4} q(t) d t=\int_{-\infty}^{0} \frac{q d q}{q^{2}+\frac{2 k(s-1)}{1-k^{2}} \sqrt{\left(1-k^{2}\right) q^{2}+s^{2}}+\frac{s^{2}-k^{2} s(2-s)}{1-k^{2}}}=-\infty
$$


as required. Let $p( \pm \pi / 4)=0$. The extension of $p$ to $\mathbb{R}$ by the antiperiodicity relation $p(\varphi+\pi / 2)=-p(\varphi)$ is continuous. In fact, it is continuously differentiable, which will follow once we prove that $p^{\prime}(\varphi)$ has a finite limit as $\varphi \uparrow \pi / 4$. When $\varphi$ is sufficiently close to $\pi / 4$, we have $q(\varphi)<-1$, and so

$$
\begin{aligned}
\log \left(-p^{\prime}(\varphi)\right)=\log p(\varphi)+\log (-q(\varphi))=\int_{0}^{\varphi} q(t) d t-\int_{q(\varphi)}^{-1} \frac{d t}{t} \\
\quad=\int_{q(\varphi)}^{0}\left\{\frac{q}{q^{2}+\frac{2 k(s-1)}{1-k^{2}} \sqrt{\left(1-k^{2}\right) q^{2}+s^{2}}+\frac{s^{2}-k^{2} s(2-s)}{1-k^{2}}}-\frac{\chi_{\{q<-1\}}}{q}\right\} d q .
\end{aligned}
$$

The last integral converges as $\varphi \uparrow \pi / 4$ because the integrand is $O\left(q^{-2}\right)$ as $q \rightarrow$ $-\infty$. Thus $p^{\prime}$ is continuous at $\pm \pi / 4$.

Later we will see that $p^{\prime \prime}$ is not defined at the points $\pi / 4+\pi n / 2, n \in \mathbb{Z}$. However, it is continuous and uniformly bounded outside of those points. To prove this it is sufficient to consider $p$ on the interval $(-\pi / 4, \pi / 4)$. Multiplying (4.2) by $p$ and using the identity $p^{\prime \prime}=p\left(q^{\prime}+q^{2}\right)$, we obtain

$$
p^{\prime \prime}(\varphi)=-\frac{2 k(s-1)}{1-k^{2}} \sqrt{\left(1-k^{2}\right) p^{\prime}(\varphi)^{2}+s^{2} p(\varphi)^{2}}-\frac{s^{2}-k^{2} s(2-s)}{1-k^{2}} p(\varphi)
$$

for all $\varphi \in(-\pi / 4, \pi / 4)$. Since $p$ and $p^{\prime}$ are uniformly bounded, so is $p^{\prime \prime}$. In particular, it follows that $p^{\prime}$ is absolutely continuous and $p^{\prime \prime}$ is its distributional derivative.

Another consequence of (4.4) is that $p$ is concave on $(-\pi / 4, \pi / 4)$. This in turn implies that $p^{\prime}$ does not vanish at $\pm \pi / 4$. Using (4.4) again, we see that

$$
\lim _{|\varphi| \uparrow \pi / 4} p^{\prime \prime}(\varphi)=-\frac{2 k(s-1)}{\sqrt{1-k^{2}}}\left|p^{\prime}(\pi / 4)\right|<0 .
$$

Since $p$ was extended antiperiodically, it follows that $p^{\prime \prime}$ indeed has a jump discontinuity at the points $\pi / 4+\pi n / 2, n \in \mathbb{Z}$.

Finally, we consider the function $u\left(r e^{i \varphi}\right)=r^{s} p(\varphi)$. By the results of the preceding paragraph, the second-order partial derivatives of $u$ are essentially bounded in any annulus $\left\{r e^{i \varphi}: 0<r_{1} \leq r \leq r_{2}<\infty\right\}$. Since they are also homogeneous of degree $s-2>-1$, we conclude that $u \in W_{\text {loc }}^{2,2}(\mathbb{C})$. Now the mapping $f=\partial u / \partial z$ is in $W_{\text {loc }}^{1,2}(\mathbb{C} ; \mathbb{C})$. Furthermore, $p$ satisfies (3.10) with equality and with $\beta=s$ and $\zeta=s^{2}$. To see this, use (4.3) and the identity $p\left(q^{\prime}+q^{2}\right)=p^{\prime \prime}$. The equivalence of (3.10) and (1.4) shows that $f$ is a $K$-quasiregular mapping. This completes our construction.

Remark 4.2. The value of $\alpha_{K}$ given by (3.2) is strictly smaller than $\alpha_{K}^{(1)}$ defined by (1.7). This can be verified by direct computations. Alternatively, consider the 
$K$-quasiregular gradient mapping

$$
f(z)=|z|^{\alpha}\left(\frac{z}{|z|}-\frac{1-\alpha}{3+\alpha} \frac{|z|^{3}}{z^{3}}\right), \quad \alpha=\alpha_{K}^{(1)},
$$

introduced in [28]. According to [28], $f(z)=\partial u / \partial z$, where

$$
u(z)=\frac{4}{\alpha+3}|z|^{\alpha-1} \operatorname{Re}\left(z^{2}\right) .
$$

Applying Theorem 3.1 to $u$, we obtain $\alpha_{K}^{(1)} \geq \alpha_{K}$. The possibility that $\alpha_{K}^{(1)}=\alpha_{K}$ can be excluded by carrying out the computations in the proof of Theorem 3.1 with $u$ given by (4.6).

A common way of constructing counterexamples in the theory of PDE is to first define a $C^{\infty}$ function on the unit sphere and then extend it to the unit ball so that the extension is smooth everywhere except at the origin (e.g. [21, 33, 40, 46, 48]). In contrast to this, the function $u\left(r e^{i \varphi}\right)=r^{s} p(\varphi)$ provided by Theorem 4.1 is not even $C^{2}$ away from the origin. More precisely, $u\left(r e^{i \varphi}\right)$ is $C^{\infty}$ in each of the four sectors

$$
\left\{r e^{i \varphi}: r>0,|\varphi-\pi n / 2|<\pi / 4\right\}, \quad n=0, \ldots, 3 .
$$

In each of these sectors either $\mathcal{P}^{+}\left(D^{2} u\right)=0$ or $\mathcal{P}^{-}\left(D^{2} u\right)=0$, where $\mathcal{P}^{+}$and $\mathcal{P}^{-}$ are Pucci's extremal operators $[12,41]$. On the boundary of each sector $u$ vanishes and its second derivative in $\varphi$ has a jump discontinuity, according to the proof of Theorem 4.1.

We can compute the complex dilatation $\mu$ of $f=\partial u / \partial z$ as in (3.9).

$$
\mu(\varphi)=\frac{\partial f / \partial \bar{z}}{\partial f / \partial z}\left(r e^{i \varphi}\right)=-e^{2 i \varphi} \frac{p^{\prime \prime}(\varphi)+s^{2} p(\varphi)}{p^{\prime \prime}(\varphi)+s(2-s) p(\varphi)+2 i(s-1) p^{\prime}(\varphi)} .
$$

Following [41] and [32], define the matrix-valued function

$$
B\left(r e^{i \varphi}\right)=\left(\begin{array}{cc}
1-\operatorname{Re} \mu(\varphi) & \operatorname{Im} \mu(\varphi) \\
\operatorname{Im} \mu(\varphi) & 1+\operatorname{Re} \mu(\varphi)
\end{array}\right), \quad r>0, \varphi \in \mathbb{R} .
$$

According to [32, 41], $u$ is a strong solution of the equation

$$
\operatorname{Tr}\left(B(z) D^{2} u(z)\right)=0 \text {, a.e. } z \in \mathbb{C} .
$$

Like the Gilbarg-Serrin operators mentioned in $\S 1, \operatorname{Tr}\left(B(z) D^{2}\right)$ has coefficients independent of $r$. Unlike the Gilbarg-Serrin operators, it has coefficients with a large set of discontinuity, namely $\left\{z \in \mathbb{C}: \operatorname{Re}\left(z^{2}\right)=0\right\}$. We hope that our construction of the "extremal" operator $\operatorname{Tr}\left(B(z) D^{2}\right)$ and the structure of its discontinuity set can provide some ideas for the treatment of higher-dimensional equations in non-divergence form. 


\section{Self-similar mappings}

Self-similar mappings, defined by (5.1) below, provide a natural generalization of homogeneous mappings. It is therefore of interest to extend Theorem 3.1 to this class of quasiregular gradient mappings. Although we have been unable to do this, we can derive an asymptotically sharp estimate for the order of vanishing of selfsimilar mappings. The proof of this estimate is quite different from what we did in the two preceding sections.

Theorem 5.1. Let $f$ be a $K$-quasiregular gradient mapping, $K>1$, and suppose that there is $\Lambda>1$ and $\alpha \in(0,1)$ such that

$$
f(\Lambda z)=\Lambda^{\alpha} f(z), \quad z \in \mathbb{C} .
$$

Then $\alpha \geq \alpha_{K}^{(3)}$, where $\alpha_{K}^{(3)} \in(0,1)$ is determined from the equation

$$
\frac{(1-\alpha)(3+\alpha)}{\sqrt{9+22 \alpha^{2}+\alpha^{4}}}=\frac{K-1}{K+1} .
$$

Remark 5.2. Note that $\alpha_{K}^{(3)} \approx 3 / K \approx \alpha_{K}$ for large $K$, where $\alpha_{K}$ is defined by (3.2). Since homogeneous mappings are self-similar, Theorem 4.1 shows that Theorem 5.1 is asymptotically sharp.

Proof. Since $r^{-\alpha} f\left(r e^{i \varphi}\right)$ is a doubly-periodic function of $\log r$ and $\varphi$, it can be expanded into a double Fourier series

$$
f\left(r e^{i \varphi}\right)=\sum_{m, n \in \mathbb{Z}} c_{m n} r^{\alpha+i m \gamma} e^{i n \varphi},
$$

where $\gamma=2 \pi / \log \Lambda$. Since the first-order derivatives of $f$ are square integrable, we can differentiate (5.2) to obtain Fourier expansions for $\partial f / \partial z$ and $\partial f / \partial \bar{z}$ that converge in the $L^{2}$ sense. Specifically,

$$
\begin{aligned}
& \frac{\partial f}{\partial z}=\frac{1}{2} \sum_{m, n \in \mathbb{Z}}(\alpha+n+i m \gamma) c_{m n} r^{\alpha-1+i m \gamma} e^{i(n-1) \varphi} ; \\
& \frac{\partial f}{\partial \bar{z}}=\frac{1}{2} \sum_{m, n \in \mathbb{Z}}(\alpha-n+i m \gamma) c_{m n} r^{\alpha-1+i m \gamma} e^{i(n+1) \varphi} .
\end{aligned}
$$

Now inequality (1.4) and Parseval's formula imply

$$
\sum_{m, n \in \mathbb{Z}}\left\{(\alpha-n)^{2}+(m \gamma)^{2}\right\}\left|c_{m n}\right|^{2} \leq k^{2} \sum_{m, n \in \mathbb{Z}}\left\{(\alpha+n)^{2}+(m \gamma)^{2}\right\}\left|c_{m n}\right|^{2} .
$$

Recall that $\partial f / \partial \bar{z}$ is real-valued, which is equivalent to

$$
(\alpha-n+i m \gamma) c_{m n}=(\alpha+n+2+i m \gamma) \bar{c}_{-m,-n-2}, \quad m, n \in \mathbb{Z} .
$$


Combining (5.3) and (5.4), we obtain

$$
\begin{aligned}
& \sum_{m \in \mathbb{Z}}\left\{(\alpha+1)^{2}+(m \gamma)^{2}\right\}\left|c_{m,-1}\right|^{2}+2 \sum_{\substack{m \in \mathbb{Z} \\
n \geq 0}}\left\{(\alpha-n)^{2}+(m \gamma)^{2}\right\}\left|c_{m n}\right|^{2} \\
& \leq k^{2} \sum_{m \in \mathbb{Z}}\left\{(\alpha-1)^{2}+(m \gamma)^{2}\right\}\left|c_{m,-1}\right|^{2} \\
& \quad+k^{2} \sum_{\substack{m \in \mathbb{Z} \\
n \geq 0}}\left\{(\alpha+n)^{2}+(m \gamma)^{2}+\frac{(n+2-\alpha)^{2}+(m \gamma)^{2}}{(n+2+\alpha)^{2}+(m \gamma)^{2}}\left[(\alpha-n)^{2}+(m \gamma)^{2}\right]\right\}\left|c_{m n}\right|^{2} .
\end{aligned}
$$

Let $m, n \in \mathbb{Z}$ be such that the multiplier of $\left|c_{m n}\right|^{2}$ on the left-hand side of the last inequality is majorized by its counterpart on the right-hand side. It is easy to see that $n \neq 0,-1$. Therefore,

$$
\begin{aligned}
\frac{2}{k^{2}}\left\{(n-\alpha)^{2}+(m \gamma)^{2}\right\} \leq & (n+\alpha)^{2}+(m \gamma)^{2} \\
& +\frac{(n+2-\alpha)^{2}+(m \gamma)^{2}}{(n+2+\alpha)^{2}+(m \gamma)^{2}}\left[(n-\alpha)^{2}+(m \gamma)^{2}\right]
\end{aligned}
$$

for some $n \geq 1$ and $m \in \mathbb{Z}$. Let $\xi=(m \gamma)^{2}$. Then

$$
\frac{2}{k^{2}} \leq \frac{(n+\alpha)^{2}+\xi}{(n-\alpha)^{2}+\xi}+\frac{(n+2-\alpha)^{2}+\xi}{(n+2+\alpha)^{2}+\xi}=: F(n, \xi) .
$$

We claim that $\sup \{F(t, \xi): t \geq 1, \xi \geq 0\}=F(1,0)$.

It is easy to prove [28] that $\sup \{F(t, 0): t \geq 1\}=F(1,0)>2$. Fix $t \geq 1$ and consider the function $\xi \mapsto F(t, \xi), \xi \geq 0$. Since $\lim _{\xi \rightarrow \infty} F(t, \xi)=2$, the claim will follow once we prove that there are no local maxima for $\xi \in(0, \infty)$. The partial derivative

$$
\frac{\partial F}{\partial \xi}=\frac{4 \alpha(t+2)}{\left((t+2+\alpha)^{2}+\xi\right)^{2}}-\frac{4 \alpha t}{\left((t-\alpha)^{2}+\xi\right)^{2}}
$$

vanishes when

$$
(t+2+\alpha)^{2}+\xi=\left((t-\alpha)^{2}+\xi\right) \sqrt{1+2 / t},
$$

that is, for at most one value of $\xi$. Since

$$
\lim _{\xi \rightarrow \infty} \xi^{2} \frac{\partial F}{\partial \xi}=8 \alpha>0
$$

it follows that the function $\xi \mapsto F(t, \xi)$ does not have a local maximum at any point $\xi \in(0, \infty)$. Thus

$$
\sup \{F(t, 0): t \geq 1, \xi \geq 0\}=F(1,0)=2 \frac{9+22 \alpha^{2}+\alpha^{4}}{(1-\alpha)^{2}(3+\alpha)^{2}}
$$


which by (5.5) implies

$$
k \geq \frac{(1-\alpha)(3+\alpha)}{\sqrt{9+22 \alpha^{2}+\alpha^{4}}} .
$$

Since the right-hand side is decreasing in $\alpha$ [28], the theorem follows.

Remark 5.3. The self-similarity assumption (5.1) can be weakened to allow "similarity with rotation". More precisely, it can be replaced with $f(\Lambda z)=\Lambda^{\alpha+i \tilde{\alpha}} f(z)$ for some $\Lambda>1, \alpha \in(0,1)$ and $\tilde{\alpha} \in \mathbb{R}$. The above argument then applies with $m \gamma$ replaced by $m \gamma+\tilde{\alpha}$.

Theorem 5.1 provides additional evidence that the optimal Hölder exponent for general strong solutions of (1.1) is equal to the exponent $\alpha_{K}$ in Theorem 3.1.

\section{Concluding remarks}

In this paper we considered only Hölder regularity of solutions. One can also ask about the sharp degree of regularity on the scale of Sobolev spaces. A celebrated theorem of Astala [2] says that every $K$-quasiregular mapping in the plane is locally in $W^{1, p}$ for every $p<p_{K}:=2 K /(K-1)$, but does not necessarily belong to $W_{\text {loc }}^{1, p_{K}}$. Consequently, every strong solution of (1.1) is locally in $W^{2, p}$ for every $p<p_{K}$. Astala, Faraco and Székelyhidi [3] used convex integration to construct strong solutions of (1.1) that do not belong to $W_{\text {loc }}^{2, p_{K}}$. This means that quasiregular gradient mappings do not possess any higher Sobolev regularity than general quasiregular mappings.

By the Morrey embedding theorem, $W^{1, p} \subset C^{0, \alpha}$ with $\alpha=1-2 / p$ provided that $p>2$. Let us say that exponents $p$ and $\alpha$ are coupled if $\alpha=1-2 / p$. For the divergence form equations in the plane the optimal values of $p$ and $\alpha$ are $p=2 \sqrt{K} /(\sqrt{K}-1)$ (not attained) and $\alpha=1 / \sqrt{K}$ (attained), see [2, 3, 30, 40]. These values are coupled in the above sense. By contrast, Theorem 1.1 together with the results in [3] show that the optimal Hölder and Sobolev exponents for the non-divergence form equations are not coupled.

\section{References}

[1] L. V. AhLFors, On quasiconformal mappings, J. Anal. Math. 3 (1954), 1-58.

[2] K. Astala, Area distortion of quasiconformal mappings, Acta Math. 173 (1994), 37-60.

[3] K. Astala, D. Faraco and L. SzÉKelyhidi JR., Convex integration and the $L^{p}$ theory of elliptic equations, Max Planck Institute MIS, preprint no. 70 (2004).

[4] K. Astala, T. Iwaniec and G. Martin, Pucci's conjecture and the Alexandrov inequality for elliptic PDEs in the plane, to appear in J. Reine Angew. Math.

[5] K. Astala, T. Iwaniec and G. Martin, "Elliptic partial differential equations and quasiconformal mappings in the plane", monograph in preparation.

[6] K. Astala, T. Iwaniec and E. Saksman, Beltrami operators in the plane, Duke Math. J. 107 (2001), 27-56. 
[7] P. BÉNILAN and F. BOUHSISS, Une remarque sur l'unicité des solutions pour l'opérateur de Serrin, C. R. Acad. Sci. Paris Sér. I Math. 325 (1997), 611-616.

[8] L. BERS, "Mathematical aspects of subsonic and transonic gas dynamics", Surveys in Applied Math., vol. 3, John Wiley \& Sons, New York, 1958.

[9] L. BERS and M. SCHECHTER, Elliptic equations, In: "Partial Differential Equations" (Proc. Summer Seminar, Boulder, Colorado, 1957), 131-299; Interscience, New York, 1964.

[10] B. Bojarski, Subsonic flow of compressible fluid, Arch. Mech. (Arch. Mech. Stos.) 18 (1966), 497-520.

[11] P. BUOnocore and P. MANSElli, Nonunique continuation for plane uniformly elliptic equations in Sobolev spaces, Ann. Scuola Norm. Sup. Pisa Cl. Sci. (4) 29 (2000), 731-754.

[12] L. A. CAFFARElli and X. CABrÉ, "Fully nonlinear elliptic equations", AMS Colloquium Publications, vol. 43. AMS, Providence, 1995.

[13] L. A. CAFFARelli, E. B. FABes and C. E. Kenig, Completely singular elliptic-harmonic measures, Indiana Univ. Math. J. 30 (1981), 917-924.

[14] F. Chiarenza, M. Frasca and P. Longo, $W^{2, p}$-solvability of the Dirichlet problem for nondivergence elliptic equations with VMO coefficients, Trans. Amer. Math. Soc. 336 (1993), 841-853.

[15] L. D'ONOFRIO and L. GRECO, On the regularity of solutions to a nonvariational elliptic equation, Ann. Fac. Sci. Toulouse Math. (6) 11 (2002), 47-56.

[16] L. C. Evans and R. F. GARIEPY, "Measure theory and fine properties of functions", CRC Press, Boca Raton, 1992.

[17] R. FINN and J. SERRIN, On the Hölder continuity of quasiconformal and elliptic mappings, Trans. Amer. Math. Soc. 89 (1958), 1-15.

[18] C. GiannotTi, A compactly supported solution to a three-dimensional uniformly elliptic equation without zero-order term, J. Differential Equations 201 (2004), 234-249.

[19] D. GILBARG and J. SERRIN, On isolated singularities of solutions of second order elliptic differential equations, J. Anal. Math. 4 (1955/56), 309-340.

[20] D. Gilbarg and N. S. Trudinger, "Elliptic partial differential equations of second order", 2nd ed. Springer-Verlag, Berlin-Heidelberg-New York, 1983.

[21] L. GReCo and C. SBordone, Sharp upper bounds for the degree of regularity of the solutions to an elliptic equation, Comm. Partial Differential Equations 27 (2002), 945-952.

[22] P. Hartman, Hölder continuity and non-linear elliptic partial differential equations, Duke Math. J. 25 (1958), 57-65.

[23] T. IWANIEC and J. J. MANFREDI, Regularity of p-harmonic functions on the plane, Rev. Mat. Iberoamericana 5 (1989), no. 1-2, 1-19.

[24] T. IWANIEC and G. MARTIN, "Geometric function theory and nonlinear analysis", Oxford Univ. Press, Oxford-New York, 2001.

[25] R. R. JENSEN, Uniformly elliptic PDEs with bounded, measurable coefficients, J. Fourier Anal. Appl. 2 (1996), 237-259.

[26] C. E. Kenig, Potential theory of non-divergence form elliptic equations, In: "Dirichlet forms", 89-128, Lecture Notes in Math., vol. 563, Springer-Verlag, Berlin-HeidelbergNew York, 1993.

[27] C. E. KenIG, "Harmonic analysis techniques for second order elliptic boundary value problems", CBMS Regional Conference Series in Mathematics, vol. 83, AMS, Providence, 1994.

[28] L. V. KovAlEV and D. OpĚLA, Quasiregular gradient mappings and strong solutions of elliptic equations, In: "The p-harmonic Equation and Recent Advances in Analysis", P. Poggi-Corradini (ed.), Contemporary Math., vol. 370, AMS, Providence.

[29] O. A. LADYZHENSKAYA and N. N. URAL' TSEVA, "Linear and quasilinear elliptic equations", Academic Press, New York-London, 1968.

[30] F. LEONETTI and V. NESI, Quasiconformal solutions to certain first order systems and the proof of a conjecture of G. W. Milton, J. Math. Pures Appl. (9) 76 (1997), 109-124.

[31] V. A. LiskeVICH, On $C^{0}$-semigroups generated by elliptic second order differential expressions on $L^{p}$-spaces, Differential Integral Equations 9 (1996), 811-826. 
[32] J. J. MAnfRedi, p-harmonic functions in the plane, Proc. Amer. Math. Soc. 103 (1988), 473-479.

[33] N. G. Meyers, An $L^{p}$-estimate for the gradient of solutions of second order elliptic divergence equations, Ann. Scuola Norm. Sup. Pisa Cl. Sci. (3) 17 (1963), 189-206.

[34] C. B. Morrey, On the solutions of quasi-linear elliptic partial differential equations, Trans. Amer. Math. Soc. 43 (1938), 126-166.

[35] C. B. MorRey, "Multiple Integrals in the Calculus of Variations", Springer-Verlag, BerlinHeidelberg-New York, 1966.

[36] N. NADIRASHVILI, Nonuniqueness in the martingale problem and the Dirichlet problem for uniformly elliptic operators, Ann. Scuola Norm. Sup. Pisa Cl. Sci. (4) 24 (1997), 537549.

[37] L. Nirenberg, On nonlinear elliptic partial differential equations and Hölder continuity, Comm. Pure Appl. Math. 6 (1953), 103-156.

[38] L. Nirenberg, On elliptic partial differential equations, Ann. Scuola Norm. Sup. Pisa Cl. Sci. (3) 13 (1959), 115-162.

[39] S. Petermichl and A. Volberg, Heating of the Ahlfors-Beurling operator: weakly quasiregular maps on the plane are quasiregular, Duke Math. J. 112 (2002), 281-305.

[40] L. C. PICCININI and S. SPAGNOLO, On the Hölder continuity of solutions of second order elliptic equations in two variables, Ann. Scuola Norm. Sup. Pisa Cl. Sci. (3) 26 (1972), 391-402.

[41] C. PUCCI, Un problema variazionale per $i$ coefficienti di equazioni differenziali di tipo ellittico, Ann. Scuola Norm. Sup. Pisa Cl. Sci. (3) 16 (1962), 159-172.

[42] C. PuCCI, Limitazioni per soluzioni di equazioni ellittiche, Ann. Mat. Pura Appl. (4) 74 (1966), 15-30.

[43] YU. G. RESHETNYAK, "Space mappings with bounded distortion", Translations of Mathematical Monographs, vol. 73. AMS, Providence, 1989.

[44] T. RICCIARDI, A sharp Hölder estimate for elliptic equations in two variables, Proc. Roy. Soc. Edimburgh 135/A (2005), 165-173.

[45] S. RicKMAN, "Quasiregular mappings", Springer-Verlag, Berlin-Heidelberg -New York, 1993.

[46] M. V. SAFONOV, Unimprovability of estimates of Hölder constants for solutions of linear elliptic equations with measurable coefficients, Math. USSR-Sb. 60 (1988), 269-281.

[47] M. V. SAFONOV, Nonuniqueness for second-order elliptic equations with measurable coefficients, SIAM J. Math. Anal. 30 (1999), 879-895.

[48] J. SERrIn, Pathological solutions of elliptic differential equations, Ann. Scuola Norm. Sup. Pisa Cl. Sci. (3) 18 (1964), 385-387.

[49] G. TAlenti, Equazioni lineari ellittiche in due variabili, Matematiche (Catania) 21 (1966), 339-376.

[50] K. O. Widman, On the Hölder continuity of solutions of elliptic partial differential equations in two variables with coefficients in $L^{\infty}$, Comm. Pure. Appl. Math. 22 (1969), 669682.

[51] A. Zygmund, “Trigonometric Series”, 3rd ed., Cambridge Univ. Press, Cambridge, 2002.

Department of Mathematics

Washington University

Saint Louis, Missouri 63130, USA

al@math.wustl.edu

Department of Mathematics

Washington University

Saint Louis, Missouri 63130, USA

lkovalev@math.wustl.edu 